\title{
The Hubble diagram for a system within dark energy: the location of the zero-gravity radius and the global Hubble rate (Research Note)
}

\author{
P. Teerikorpi ${ }^{1}$ and A. D. Chernin ${ }^{1,2}$ \\ 1 Tuorla Observatory, Department of Physics and Astronomy, University of Turku, 21500 Piikkiö, Finland \\ e-mail: pekkatee@utu.fi \\ 2 Sternberg Astronomical Institute, Moscow University, Moscow 119899, Russia \\ Received 3 March 2010 / Accepted 18 April 2010
}

ABSTRACT

\begin{abstract}
Aims. Here we continue to discuss the principle of the local measurement of dark energy using the normalized Hubble diagram describing the environment of a system of galaxies.

Methods. We calculate the present locus of test particles injected a fixed time ago ( $\sim$ the age of the universe), in the standard $\Lambda$ cosmology and for different values of the system parameters (the model includes a central point mass $M$ and a local dark energy density $\rho_{\mathrm{loc}}$ ) and discuss the position of the zero-gravity distance $R_{\mathrm{v}}$ in the Hubble diagram.

Results. Our main conclusion are: 1) when the local DE density $\rho_{\text {loc }}$ is equal to the global DE density $\rho_{\mathrm{v}}$, the outflow reaches the global Hubble rate at the distance $R_{2}=\left(1+z_{\mathrm{v}}\right) R_{\mathrm{v}}$, where $z_{\mathrm{v}}$ is the global zero-acceleration redshift ( $\approx 0.7$ for the standard model). This is also the radius of the ideal Einstein-Straus vacuole, 2) for a wide range of the local-to-global dark energy ratio $\rho_{\text {loc }} / \rho_{\mathrm{v}}$, the local flow reaches the known global rate (the Hubble constant) at a distance $R_{2} \gtrsim 1.5 \times R_{\mathrm{v}}$. Hence, $R_{\mathrm{v}}$ will be between $R_{2} / 2$ and $R_{2}$, giving upper and lower limits to $\rho_{\mathrm{loc}} / M$. For the Local Group, this supports the view that the local density is near the global one.
\end{abstract}

Key words. Local Group - dark energy - cosmological parameters

\section{Introduction}

The standard $\Lambda \mathrm{CDM}$ cosmology views dark energy as having constant density everywhere. If so, its local density should be identical to that inferred from global observations of Supernovae Ia (Riess et al. 1998; Perlmutter et al. 1999) and the CMB (Spergel et al. 2007), $\rho_{\mathrm{v}} \approx 7 \times 10^{-30} \mathrm{~g} / \mathrm{cm}$.

Dark energy can be studied locally (Chernin et al. 2006; Teerikorpi et al. 2008), as its "antigravity" can affect galaxy motions near us in a volume a few Mpc across (Chernin et al. 2003). Also, the real mass of a system can only be found if the dark energy is included, because gravitating systems "lose" a part of their gravity due to the antigravity of the dark energy within them (Chernin et al. 2009).

The homogeneity and constancy of the dark energy imply an important fact: lumps of matter, caused by gravitational instability in the expanding universe, did not appear alone, but together with zero-gravity surfaces around them. The gravity of the mass $M$ of the concentration and the repulsion of the dark energy with density $\rho_{\mathrm{v}}$ are equal at the zero-gravity radius $R_{\mathrm{v}}$ (Chernin 2001):

$R_{\mathrm{v}}=\left(\frac{3 M}{8 \pi \rho_{\mathrm{v}}}\right)^{1 / 3}$

Antigravity dominates at $R>R_{\mathrm{v}}$, and gravity is stronger than antigravity at $R<R_{\mathrm{v}}$.

This concept of the spatial border $R_{\mathrm{v}}$ does not apply to the perfectly smooth Friedmann models (these have instead the temporary border separating deceleration and acceleration everywhere), but it is important for understanding the local structure and dynamics of galaxy systems: the zero-gravity border exists during all the life-time of the system since its formation.

The two major parameters of a "local Hubble cell" are the total mass of the group or cluster and the local density of the dark energy. If the mass is, say, $2 \times 10^{12} M_{\odot}$ and the local dark energy density is equal to its global value $\rho_{\mathrm{v}}$, then $R_{\mathrm{v}}=1.3 \mathrm{Mpc}$. Or, if the zero-gravity radius is known from observations, the mass may be written as:

$M=\frac{8 \pi}{3} \rho_{\mathrm{v}} R_{\mathrm{v}}^{3} \simeq 0.9 \times 10^{12}\left[R_{\mathrm{v}} /(\mathrm{Mpc})\right]^{3} M_{\odot}$.

Thus one can determine the DE density by comparing the predicted value of the group mass to the mass known from other methods. However, this requires an independent estimate for the zero-gravity distance. As we have noted (Chernin et al. 2006), the size of the group or the zero-velocity distance is a strict lower limit to $R_{\mathrm{v}}$, giving an upper limit to the $\mathrm{DE}$ density. But how to obtain an upper limit to $R_{\mathrm{v}}$ and thus the interesting lower limit to DE? In our previous studies (Chernin et al. 2006, 2009) we have assumed that the Hubble flow begins beyond $R_{\mathrm{V}}$. Here we study this question more quantitatively and pay special attention to the distance where the local outflow reaches the global Hubble rate, as this has particular significance in the point-mass model.

\section{Position of $\boldsymbol{R}_{\mathrm{v}}$ in the Hubble diagram}

If the local DE density is equal to the global one, there is the suggestive coincidence with $R_{\mathrm{V}} \approx 1.3-1.4 \mathrm{Mpc}$ being also the distance where the local Hubble flow begins to be seen. But one 


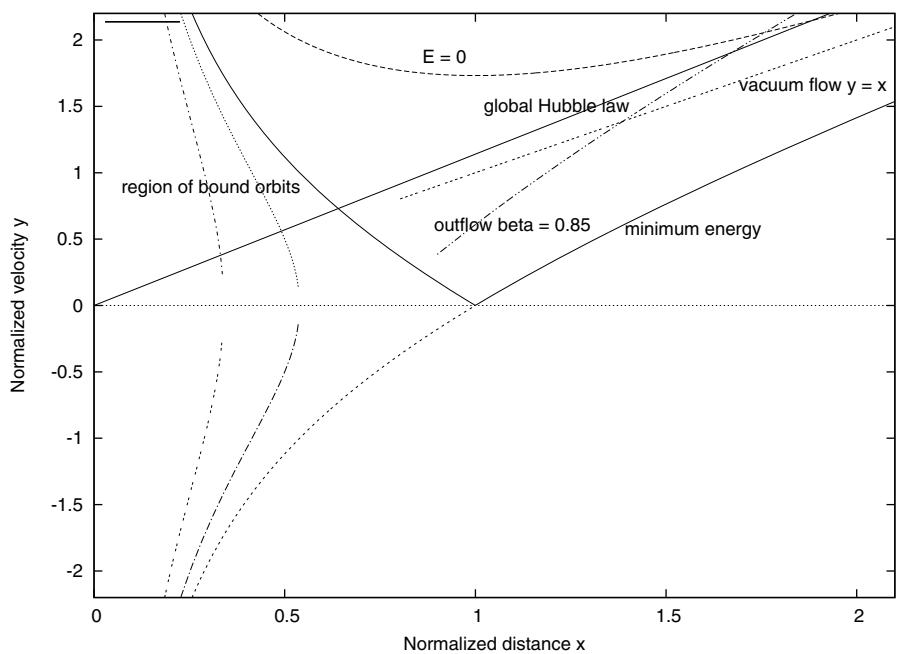

Fig. 1. The normalized distance-velocity diagram showing the predicted local flow for the age of the standard world model, corresponding to $\beta=t / T_{\mathrm{v}}=0.85$. The local flow reaches the global Hubble rate around $x \approx 1.7 R_{\mathrm{v}}$.

would like to find a way to measure the zero-gravity distance independently, using local data on the mass and the outflow. What could be its signature in the observations? In order to clarify this question we have calculated, within the two-component model, the present expected locus of outflowing dwarf galaxies for several different values of the relevant parameters.

\subsection{The normalized Hubble diagram}

Previously, we introduced the concept of a normalized Hubble diagram, in order to study the kinematic structure of a group and its surroundings (Teerikorpi et al. 2008) in the presence of dark energy. If one fixes the mass of the group and the local dark energy density, one can calculate the zero-gravity radius $R_{\mathrm{v}}$ and the vacuum Hubble constant $H_{\mathrm{v}}=\left(8 \pi G \rho_{\mathrm{v}} / 3\right)^{1 / 2}$. Then in the dimensionless representation with $R / R_{\mathrm{v}}$ and $V / H_{\mathrm{v}} R_{\mathrm{v}}$ as $x$ and $y$-axes, respectively, one may conveniently describe different dynamical regions of the system (see Fig. 1).

Writing the total mechanical energy as $E=-\alpha G M / R_{\mathrm{v}}$, there is a minimum energy curve corresponding to $\alpha=\frac{3}{2}$. Test particles ejected from the region of bound orbits $\left(R<R_{\mathrm{V}}\right)$ cannot appear below this curve for $R>R_{\mathrm{v}}$. In fact, the minimum energy curve can be used to give an upper limit to the local DE density or a lower limit to $R_{\mathrm{v}}$ which may be stricter than that obtained from the size and mass of the system.

The physical sense of this minimum energy curve is that it corresponds to ejections an infinitely long time ago. Hence, any upper limit for the age provides a still stricter lower-limit curve. Consider the vacuum Hubble time $T_{\mathrm{v}}=1 / H_{\mathrm{v}}$, where

$$
\begin{aligned}
H_{\mathrm{v}} & =\left(8 \pi G \rho_{\mathrm{v}} / 3\right)^{1 / 2} \\
& =61.0 \times\left(\rho_{\mathrm{v}} / 7 \times 10^{-30} \mathrm{~g} / \mathrm{cm}^{3}\right)^{1 / 2} \mathrm{~km} \mathrm{~s}^{-1} \mathrm{Mpc}^{-1}
\end{aligned}
$$

The vacuum Hubble time $T_{\mathrm{v}}$ is larger than the global Hubble time by the factor $\left(1+\rho_{\mathrm{m}} / \rho_{\mathrm{v}}\right)^{1 / 2}=\left(\Omega_{\mathrm{V}}\right)^{-1 / 2}$ for a flat universe and for $\rho_{\mathrm{v}}=$ the global DE density. Thus $T_{\mathrm{v}}$ is a natural upper limit for the age. In the standard model $T_{\mathrm{v}}=16 \times 10^{9} \mathrm{yrs}$ and the age of the universe $\left(13.6 \times 10^{9} \mathrm{yrs}\right)$ is about $0.85 \times T_{\mathrm{v}}$.

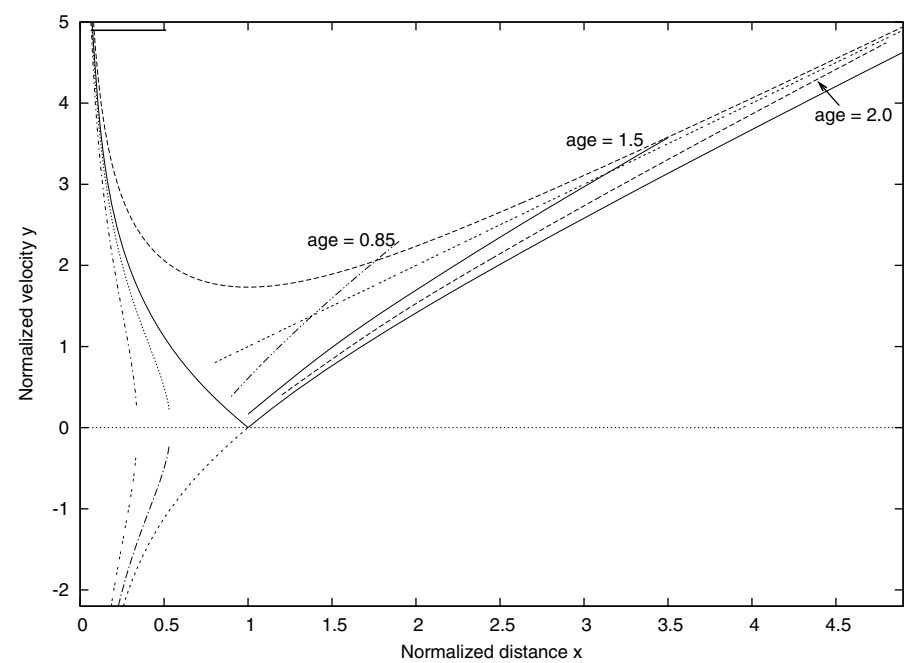

Fig. 2. The normalized distance-velocity diagram showing the predicted local flow for several flight times: $0.85,1.5$ and 2.0 vacuum Hubble times. For large distances and long times the flow approaches the vacuum flow $y=x$.

\subsection{The present distance-velocity relation}

The flight time from the center of the group $\left(x_{0} \ll 1\right)$ to the normalized distance $x=r / R_{\mathrm{v}}$, for a particle with energy $E=$ $-\alpha G M / R_{\mathrm{v}}$ can be parametrized in terms of the vacuum Hubble time $T_{\mathrm{v}}=1 / H_{\mathrm{v}}$ :

$t=T_{\mathrm{v}} \int_{x_{0}}^{x}\left(x^{2}+2 / x-2 \alpha\right)^{-1 / 2} \mathrm{~d} x$,

while the normalized velocity $y$ is related to $x$ as

$y=\left(x^{2}+2 / x-2 \alpha\right)^{1 / 2}$.

One can calculate for each location $x$ the required energy $\alpha$ for a particle to reach this distance after the flight time $t$, and thus the normalized velocity $y$. This is similar to what has been done in Lemaître-Tolman solutions (e.g. Peirani \& de Freitas Pacheco 2006, 2008).

As an example, in the normalized diagram of Fig. 1 we have indicated the predicted current distance-velocity curve for the age $t / T_{\mathrm{v}}=0.85$ for the case where the local DE density is equal to the global one (Eq. (5) or (7) with $\rho_{\text {loc }}=\rho_{\mathrm{v}}$ ). The straight line above the vacuum Hubble flow $(y=x)$ is the global Hubble law with $H=\left(\Omega_{\mathrm{v}}\right)^{-1 / 2} H_{\mathrm{v}}$ for $\Omega_{\mathrm{v}}=0.77$.

In Fig. 2 we illustrate how the increasing flight time $(0.85$, 1.5 , and $2.0 \times T_{\mathrm{v}}$ ) influences the distance-velocity relation in the normalized Hubble diagram. Note that at large distances and long times the flow approches the vacuum flow $y=x$. The vacuum flow is also the asymptotic line for the zero-energy and the minimum energy curves, as can be seen from Eq. (6).

From the $y=y(x)$ we can obtain the locus of present distance-velocity positions as $\left(x R_{\mathrm{v}}, y H_{\mathrm{v}} R_{\mathrm{v}}\right)$. So, fixing the mass $M$ and the DE density $\rho_{\mathrm{v}}$ (which give the distance $R_{\mathrm{v}}$ and the vacuum Hubble constant $H_{\mathrm{v}}$ needed in the normalization), and the flight time $t / T_{\mathrm{v}}$ (say, the age of the universe) we can calculate the velocity-distance relation for the ordinary Hubble diagram. For example, in Chernin et al. (2009) the mass was varied and the predicted Hubble relation was compared with the outflow around the Local Group, assuming that the local DE density is the same as the global one. 


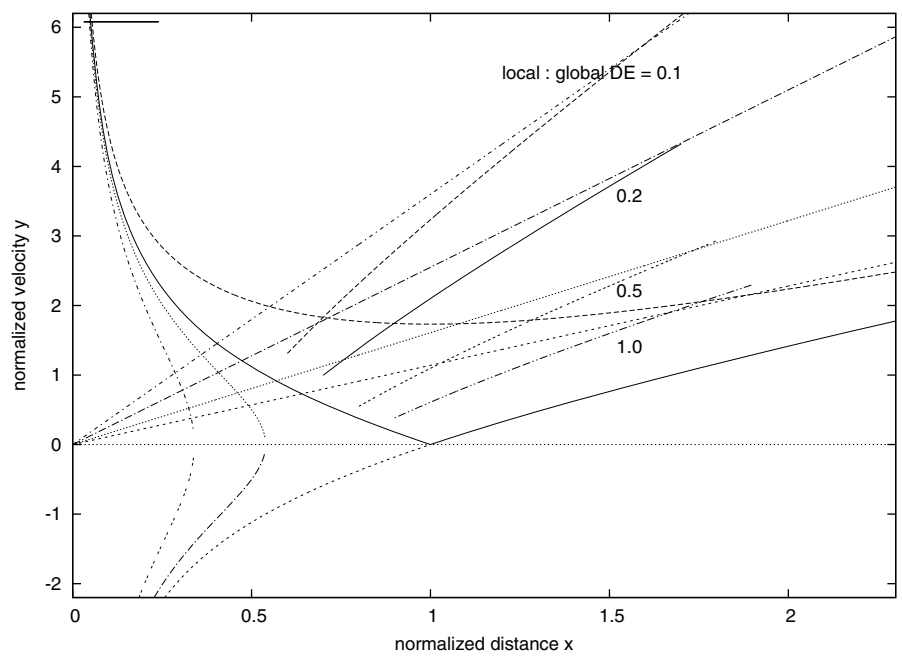

Fig. 3. The normalized distance-velocity diagram for different values of the local-to-global dark energy density ratio. Note that the predicted local flow reaches the global Hubble rate (the straight lines beginning from the origo) around 1.5-1.7 times the zero-gravity distance.

\subsection{Times spent in gravitation and $D E$ dominated regions}

We see from Fig. 2 that in the standard model the present Hubble relation deviates from the vacuum flow $y=x$ and, as mentioned, only in the distant future it approaches that relation for the $\mathrm{DE}$ dominated cosmos. In fact, the galaxies presently located in the range $1 \lesssim x \lesssim 1.7$ have spent in the past most of their time within the gravity dominated region and less than half in the DE dominated zone. For the standard model (age $=0.85$ vacuum Hubble time), it is only at $x \gtrsim 1.8$ where the galaxies have spent about equal or longer time in the DE dominated region. This is why the current Hubble relation has not had time to approach closer to the vacuum flow, even though the static zero-energy border has existed since the formation of the group.

\subsection{When the local DE density differs from the global value}

We see from Fig. 1 that the Hubble ratio of the local flow aproaches the global value around $R \approx 1.7 R_{\mathrm{v}}$. This suggests the location where one can bracket from upwards the possible values of $R_{\mathrm{v}}$. But is this criterion - where the observed local flow is close to the global rate - valid also for dark energy densities smaller than the global one?

In Eq. (5) the time $T_{\mathrm{v}}$ actually means the vacuum Hubble time corresponding to the local DE density $\rho_{\text {loc }}$. As we want to use the global vacuum Hubble time (related to the standard model) as a convenient unit also when the local and global densities differ, we write:

$t / T_{\mathrm{v}}=\left(\rho_{\mathrm{v}} / \rho_{\mathrm{loc}}\right)^{1 / 2} \int_{x_{0}}^{x}\left(x^{2}+2 / x-2 \alpha\right)^{-1 / 2} \mathrm{~d} x$.

In the normalized diagram of Fig. 3 we show the calculated local Hubble relations for several cases of the local-to-global DE ratios, $\rho_{\text {loc }} / \rho_{\mathrm{v}}=1,0.5,0.2$, and 0.1 . For each curve we show the corresponding global Hubble line (the same in ordinary units, but differing in the normalized presentation).

It is seen that in each case the local flow approaches the global Hubble law well beyond the zero-gravity radius $R_{\mathrm{v}}$, in fact at about the same normalized $x$-location. In the ordinary Hubble diagram these locations may differ much (due to the different values of $R_{\mathrm{v}}$ ), but it is important that the criterion which we have used in our previous papers (e.g. Chernin et al. 2006, 2009) thus obtains quantitative support: the known global Hubble law allows one to define a robust upper limit to $R_{\mathrm{v}}$ from the local expansion flow.

\section{Concluding remarks}

It is interesting to realize that the mass concentrations have had around them the zero-gravity surface since the formation of the concentration ${ }^{1}$.

Beyond this surface the acceleration is positive, while within it there is deceleration. We cannot directly measure the accelerations in order to define the location of the zero-gravity distance $R_{\mathrm{V}}$ and hence determine the quantity $\rho_{\text {loc }} / M$ (and hence the dark energy density, if the mass $M$ is known). However, as we have previously argued and in more detail shown here, we can obtain information about $R_{\mathrm{v}}$ by comparing the current behaviour of the local flow with the global Hubble law. The latter is related to the global time scale and is known from various large-scale determinations.

\subsection{The local outflow and the zero-acceleration redshift $z_{\mathrm{V}}$}

The calculations show that the local flow should reach the global expansion rate around a distance 1.5-1.7 times the zero-gravity radius (for the local DE density from 0.1 to 1 global density). This allows one to set an upper limit $R_{2}$ to $R_{\mathrm{v}}$ and hence a lower limit to $\rho_{\text {loc }} / M$. If the mass-point model is inadequate, so that in addition to the known mass $M$ of the group itself there is some unknown extended mass $\Delta M$ up to $R_{\mathrm{v}}$, then the lower limit to $\rho_{\text {loc }}$ from Eq. (2) is just made more robust: $\rho_{\text {loc }} / \rho_{\mathrm{v}}>[(M+$ $\Delta M)] / 0.9 R_{2}^{3}>M / 0.9 R_{2}^{3}$ (in units of $10^{12} M_{\odot}$ and Mpc).

What happens at larger distances depends on whether the local model is still valid - one finally reaches the distance $R_{\mathrm{M}}$ which gives the size of the volume from which the mass $M$ has been gathered during the formation of the group (so-called Einstein-Straus vacuole; Chernin et al. 2006). One can calculate the present radius $R_{\mathrm{M}}$ of the region from which the mass $M$ was gathered, assuming the present average cosmic mass density $\rho_{\mathrm{m}}$. It is $R_{\mathrm{M}}=\left(2 \rho_{\text {loc }} / \rho_{\mathrm{m}}\right)^{1 / 3} R_{\mathrm{v}}$ (e.g. $=1.7 R_{\mathrm{v}}$ for $\Omega_{\mathrm{v}} / \Omega_{\mathrm{m}}=$ $0.77 / 0.23)$.

Interestingly, this is the same 1.7 as in the global scale factor ratio leading to $z_{\mathrm{v}}=0.7$. Namely, the requirement that the global acceleration is zero when $\rho_{\mathrm{v}}=0.5 \rho_{\mathrm{m}}\left(z_{\mathrm{v}}\right)$ leads in terms of the current mass density to the condition $2 \rho_{\mathrm{v}}=\left(1+z_{\mathrm{v}}\right)^{3} \rho_{\mathrm{m}}$ or $\left(2 \rho_{\text {loc }} / \rho_{\mathrm{m}}\right)^{1 / 3}=\left(1+z_{\mathrm{v}}\right)=1.7$. This is also the distance where the global Hubble ratio is reached, because at this point the enclosed mass is the same as for the uniform global Friedmann model, hence the expansion rate is the same.

To repeat, when the local dark energy density is equal to the global DE density, the global Hubble rate is reached at the distance $\left(1+z_{\mathrm{V}}\right) R_{\mathrm{v}}$. This distance is also the same as the radius of the ideal Einstein-Straus vacuole $R_{\mathrm{M}}$.

\subsection{An application to the Local Group}

An implication is that the best place for the study of the local dark energy using the present approach would be where the

\footnotetext{
1 The total mass of the system within $R_{\mathrm{V}}$ may vary with time after its formation because of accretion of material from outside or escape of dwarf galaxies from the system. In reality such mass variations can hardly be very significant. In any case, the value of the zero-gravity radius should follow the total mass in accordance with Eq. (1).
} 
point mass plus void model extends beyond the standard radius $R_{\mathrm{M}}=\left(2 \rho_{\text {loc }} / \rho_{\mathrm{m}}\right)^{1 / 3} R_{\mathrm{v}}$. Interestingly, this may just be the case in the Local Volume where, according to Karachentsev et al. (2009), there seems to be an average underdensity by a factor of 2 or 3 locally, and the vacuole from which the central mass was gathered, may then exceed $R_{\mathrm{M}}$ by a factor of 1.26 or 1.44 , respectively.

If the local flow reaches the global Hubble rate around the distance $R_{2}$, as defined by sufficiently accurate data, and if $R_{2} \lesssim$ $R_{\mathrm{M}}$, then robustly

$\frac{R_{2}}{2}<R_{\mathrm{v}}<R_{2}$.

For example, if we take for the Local Group $R_{2} \approx 2.4 \mathrm{Mpc}$ and $M=2 \times 10^{12} M_{\odot}$ (Karachentsev et al. 2009), then we obtain using Eq. (2)

$0.5<\rho_{\text {loc }} / \rho_{\mathrm{v}}<2.0$.

Expressed in another way, for $M=2.0-3.5 \times 10^{12} M_{\odot}$ (see Chernin et al. 2009) and $\rho_{\mathrm{loc}}=\rho_{\mathrm{v}}$, the zero-gravity radius is 1.3 to $1.55 \mathrm{Mpc}$ and the predicted distance where the local flow reaches the global Hubble rate is 2.2-2.6 Mpc, which agrees with the observations (Karachentsev et al. 2009). Hence, as in our earlier estimations, the value of the local density proves to be near the global one, supporting the universal nature of the dark energy having the same density both globally and locally.

Acknowledgements. A.C. thanks the RFBR for partial support via the grant 10-02-00178. We also thank the anonymous referee for useful comments.

\section{References}

Chernin A. D. 2001, Physics-Uspekhi, 44, 1099

Chernin, A. D., Teerikorpi, P., \& Baryshev, Yu. V. 2003, Publ. Adv. Space Res., 31,459

Chernin, A., Teerikorpi, P., \& Baryshev, Yu. 2006, A\&A, 456, 13

Chernin, A. D., Teerikorpi, P., Valtonen, M. J., et al. 2009, A\&A, 507, 1271

Karachentsev, I. D., Kashibadze, O. G., Makarov, D. I., \& Tully, R. B. 2009, MNRAS, 393, 1265

Peirani, S., \& de Freitas Pacheco, J. A. 2006, New Ast., 11, 325

Peirani, S., \& de Freitas Pacheco, J. A. 2008, A\&A, 488, 845

Perlmutter, S., Aldering, G., Goldhaber, G., et al. 1999, ApJ, 517, 565

Riess, A. G., Filippenko, A. V., Challis, P., et al. 1998, AJ, 116, 1009

Spergel, D. N., Bean, R., Doré, O., et al. 2007, ApJS, 170, 377

Teerikorpi, P, Chernin, A. D., Karachentsev, I. D., \& Valtonen M. J. 2008, A\&A, 483,383 